\title{
Multiscale Combination of Physically-Based Registration and Deformation Modeling
}

\author{
L.V. Tsap, D.B. Goldgof and S. Sarkar
}

This article was submitted to Institute for Electrical and Electronics Engineers

Conference on Computer Vision and Pattern Recognition '00 Hilton Head, SC

June 13-15, 2000

U.S. Department of Energy

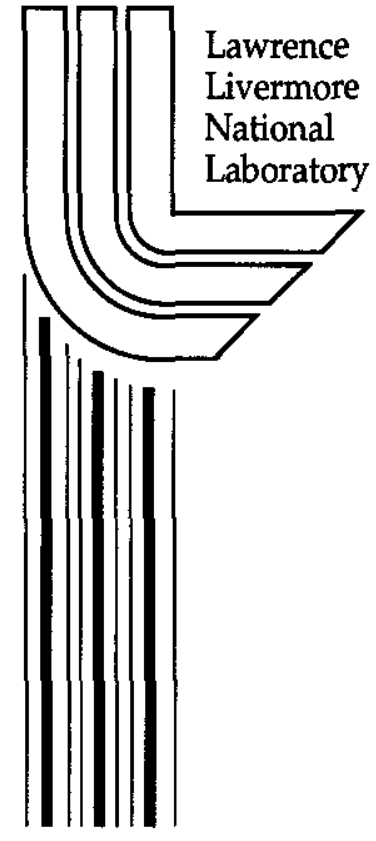

November 8, 1999 


\section{DISCLAIMER}

This document was prepared as an account of work sponsored by an agency of the United States Government. Neither the United States Government nor the University of California nor any of their employees, makes any warranty, express or implied, or assumes any legal liability or responsibility for the accuracy, completeness, or usefulness of any information, apparatus, product, or process disclosed, or represents that its use would not infringe privately owned rights. Reference herein to any specific commercial product, process, or service by trade name, trademark, manufacturer, or otherwise, does not necessarily constitute or imply its endorsement, recommendation, or favoring by the United States Government or the University of California. The views and opinions of authors expressed herein do not necessarily state or reflect those of the United States Government or the University of California, and shall not be used for advertising or product endorsement purposes.

This is a preprint of a paper intended for publication in a journal or proceedings. Since changes may be made before publication, this preprint is made available with the understanding that it will not be cited or reproduced without the permission of the author.

This report has been reproduced

directly from the best available copy.

Available to DOE and DOE contractors from the Office of Scientific and Technical Information

P.O. Box 62, Oak Ridge, TN 37831

Prices available from (423) 576-8401 http://apollo.osti.gov/bridge/

Available to the public from the National Technical Information Service

U.S. Department of Commerce 5285 Port Royal Rd., Springfield, VA 22161 http://www.ntis.gov/

OR

Lawrence Livermore National Laboratory Technical Information Department's Digital Library http://www.llnl.gov/tid/Library.html 


\section{Multiscale Combination of Physically-Based Registration and Deformation Modeling}

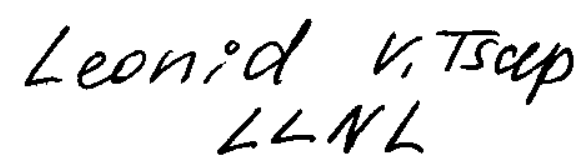

Abstract ${ }^{1}$

In this paper we present a novel multiscale approach to recovery of nonrigid motion from sequences of registered intensity and range images. The main idea of our approach is that a finite element (FEM) model can naturally handle both registration and deformation modeling using a single model-driving strategy. The method includes a multiscale iterative algorithm based on analysis of the undirected Hausdorff distance to recover correspondences. The method is evaluated with respect to speed, accuracy, and noise sensitivity. Advantages of the proposed approach are demonstrated using man-made elastic materials and human skin motion. Experiments with regular grid features are used for performance comparison with a conventional approach (separate snakes and FEM models). It is shown that the new method does not require a grid and can adapt the model to available object features.

\section{Introduction}

Nonrigid motion analysis is comprised of a large body of research directions and approaches. Nonrigid motion analysis includes establishing point correspondences necessary for tracking, estimating motion and, finally, understanding the reasons why motion occurred in the observed way and not in any other possible way. Two of the major classes of techniques for nonrigid motion analysis include snakes and finite element models.

Snakes, or active contours are energy-minimizing splines which can find and reliably track salient image contours. Snakes have specific properties used for very precise tracking, yet not related to material properties or the internal structure of the object. McEachen II and Duncan [7] tracked feature points over an entire cardiac cycle. Chandran and Potty [3] developed a strategy to avoid local minimas as a dynamic programming solution for snake energy minimization. Amini et al. [1] applied coupled B-spline snake grids to magnetic resonance images and validated results with a

\footnotetext{
${ }^{1}$ This research was supported in part by the Whitaker Foundation Biomedical Engineering Research Grant and in part by the National Science Foundation Grants IRI-9619240, EIA9729904 and CDA-9724422. This work was performed under the auspices of the U.S. Department of Energy by Lawrence Livermore National Laboratory under contract number W-7405-Eng48. UCRL-JC-XXXXXX
}

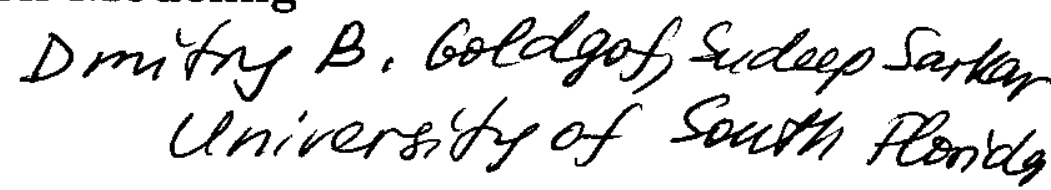

3-D cardiac motion model. Recent developments in deformable model techniques are summarized in [11].

Unlike snakes, finite element models usually include material properties of the object and precise understanding of its structure, but have no inherent way to track object's features. A variety of finite element models were proposed in the context of vision research. A finite element model that learns the correct physical model of human lips by training from real data was proposed by Basu and Pentland [2]. Martin et al. [6] employed finite element computation of analytic modes describing shape variation of structures within the human brain. Tsap et al. [13] used nonlinear finite element models to recover motion and material properties of nonrigid objects. Feature points used in finite element analysis were tracked with snakes.

Therefore, to accomplish defined motion analysis goals, it is necessary to achieve tracking similar or even better in quality than can be done with snakes, and also examine additional aspects not readily obvious from images. These features can add to the knowledge of the object (material properties, applied forces and detailed structure of the object) when using a more natural (with respect to object's properties) physically-based model, such as a finite element model that can explain the deformation process.

Most closely related works such as $[15,13,5]$ accomplished these goals using two separate models: snakes to find tag positions in images and finite element models $[15,13]$ (or similar physically-based models [5]) to compute deformation parameters (such as displacements) and strain distributions. Although FEM models produced precise solutions in terms of both displacements and strains, they utilized only information at points where tag lines intersected.

Recently, a number of hybrid approaches were developed. A framework for combining complementary techniques (registration and deformable models) was proposed by Montagnat and Delingette [8]. Another hybrid solution (2-D) based on modal analysis, employed by Tao and Huang [12], blended finiteelement-computed modes with template matching. Deformable models with parameter functions capable of adequately addressing local shape variations were proposed by Park et al. [10] and O'Donnell et al. [9]. A shape modeling approach that used multiresilution 
transformations from local to global models was introduced by Vemuri and Radisavljevic [14].

The approach proposed in this paper encompasses advantages of both techniques in a single modeldriving strategy. Both detection/tracking and accurate object model estimation are merged to provide a more comprehensive basis for nonrigid motion analysis. We propose that FEM model can naturally handle registration and modeling. Control points used for tracking are also a part of a finite element model. Therefore, not only snake intersection points (as it often occurred), but also additional tracked points are included in the model. Furthermore, the method includes a multiscale scheme based on evaluation of the undirected Hausdorff distance to speed up the process of matching features between two frames since large deformations are considered. This criteria is similar to work by Huttenlocher et al. [4]. However, in their approach, a set of image pixels in next frame formed a new model. We perform actual model transformation that simulates nonrigid motion of the object.

\subsection{Overview}

Major contributions of this work can be described along the following directions: (1) combination of registration and deformation modeling, and (2) multiscale approach to correspondence recovery. The approach assumes that a sequence of registered intensity and range images of a deforming elastic object with visible surface features (such as a grid in Fig. 4 and 6 or irregular and natural features in Section 3.4), and a physically-based model (Fig. 2(b)) are available (Section 2.1). The main idea of our approach is that a finite element model can naturally handle both registration and modeling using a single modeldriving strategy. Previously, snakes were often used to track intensity features; recovered correspondences were then incorporated into finite element models that computed deformation parameters. This work combines both approaches.

The second important aspect of the strategy is an efficient data utilization. As much available data as necessary is used. The model consists of a number of control points. The goal is defined as a correct matching of control points with grid points in the next frame. Matching occurs at different resolution levels - using 9, 49 and 217 control points (Fig. 1). The multilevel (multiscale) strategy is described in detail in Section 2.3. The matching task is similar for all scales: given the coordinates of control points, find the Hausdorff distance (defined in Section 2.2) between the model and the image (next frame), and use it to structure possible correspondences between control points and feature points in images (as discussed in Section 2.2). The selected set of correspondences drives deformation of the model.

Section 3 describes application of the proposed

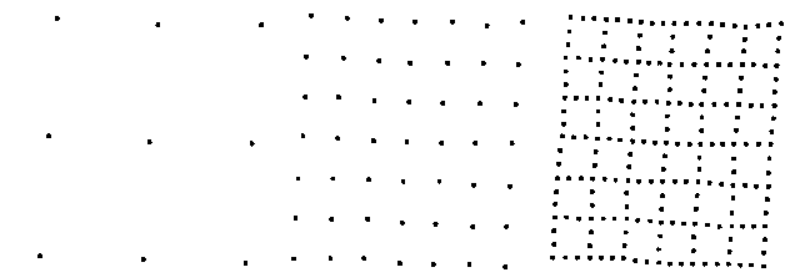

Figure 1: Configuration of control points using three different scales.

method to motion analysis of man-made elastic materials, human skin, and burn scar detection application. Objects with a grid are used for comparison with a traditional technique utilizing active contours and FEM separately. It is also shown (Section 3.4) that the proposed method does not need a grid and can take advantage of the available irregular object features or even natural features (in skin experiments). The last section summarizes the results of this research.

\section{Description of the Method}

\subsection{Data, Modeling and Assumptions}

Data acquisition, general modeling principles and necessary assumptions are discussed first. Data sequences are acquired using a K2T structured light range scanner. During acquisition, registered intensity and range images of stretching elastic objects are taken (Fig. 4 and 6). Only part of the object (elastic material or human arm) with the interest is considered. In first sets of experiments this region includes the grid which is produced with a simple stamp and aids in producing trackable features. Other sets of images contain irregular or even natural features. Let us assume that grid is separated on the intensity image (for instance, using thresholding) and the model is aligned with it. Since the data was collected initially for a different project which employed snakes, the stamp produced overlapping lines (which allows us to compare results of both approaches). For the purpose of this method they are not considered (a conventional method with snakes used original images for the performance comparison). Of course, a fully automatic method would require a different stamp. Hence, only the area bound by outside grid lines is considered (Fig. 2(a)). Therefore, the finite element model used to describe it is local. It consists of 3-D elastic shells with assigned corresponding material properties (properties of elastic materials are obtained by their mechanical testing; average skin properties are found in the recent literature). Since the geometry of the stamp is known in advance, it allows for the advance model construction necessary for the success of the method (Fig. 2(b)). Since in this research the emphasis is placed on multiscale use of control points rather than on the finite element model itself, a current set of control points (Fig. 1) from now on is referred to as our model. The method assumes alignment with the 
first frame in the sequence and consistency in point inter-relationships so that points do not overlap (occlude) each other.

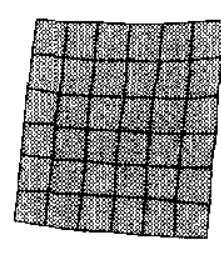

(a)

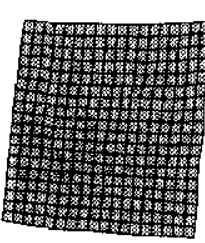

(b)

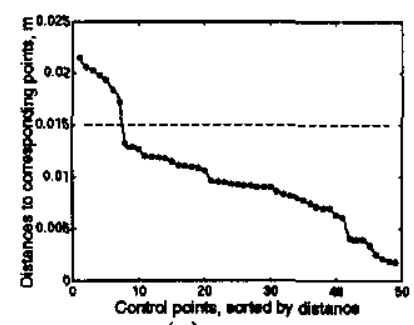

(c)
Figure 2: (a) Region of interest. (b) Finite element model. (c) Threshold $\left(T_{2}\right)$ calculation.

\subsection{Role of the Hausdorff Distance}

Control points (which are also FEM nodes or keypoints) provide a natural way to locate corresponding points in the next frame (model registration) and apply distances between them as displacements (model deformation). Control points are guided by the Hausdorff distance [4] between the model $M$ (fitted to the current frame) and the next frame in the sequence $F_{n+1}: H\left(M, F_{n+1}\right)=$ $\max \left(h\left(f\left(M, F_{n+1}\right)\right), h\left(f\left(F_{n+1}, M\right)\right)\right), \quad$ where $h\left(f\left(M, F_{n+1}\right)\right.$ is the forward distance (the distance from the model to the image) and $h\left(f\left(F_{n+1}, M\right)\right)$ is the reverse distance.

To compute the forward distance, differences are identified between each control point $m_{i}$ in the finite element model $M$ and the nearest point $a_{i}$ in the next frame $F_{n+1}$, and then the largest distance is selected: $h\left(f\left(M, F_{n+1}\right)\right)=\max _{m_{i} \in M} \min _{a_{i} \in F_{n+1}}\left\|m_{i}-a_{i}\right\|$, where $f$ denotes some transformation that occurred as a result of the motion or deformation, and $\|$.$\| is$ the Euclidean distance. The resulting control point $m_{i}$ is, therefore, the furthest control point from any range object point in $F_{n+1}$. The reverse distance $h\left(f\left(F_{n+1}, M\right)\right)$ is defined similarly.

The goal is to use the Hausdorff distance as a measure of mismatch between the model and the object, and then to reduce such differences by applying displacements to the model. This approach belongs to the class of reverse problems when the results (displacements) are given instead of the cause (body loads). As opposed to tracking with snakes (which is a separate physically-based model), the undirected Hausdorff distance can be easily combined with a finite element model. No separation into a motiondetection-oriented model and an object-propertiesoriented model is necessary. A single model is used; it is driven by the multiscale analysis of possible correspondences using the Hausdorff distance. Correspondence recovery at each step is followed by displacement calculations and their application to the model. This represents a single iteration of the method.

\subsection{Multiscale Approach to Correspon- dence Analysis and Model Deforma- tion}

Expected range of motion is addressed by the multiscale approach to correspondence analysis and model deformation. Larger motion necessitates the use of coarser alignment models before finer aspects of object deformation are addressed. Multiscale strategy discussed in this section is applicable to a large object and motion domain; however, the number of scales is based on the magnitude of size or motion and, obviously, may change for different objects.

Three scales (defined in terms of control points) are adopted for the experiments described:

1 - ICA (initial coarse alignment, 9 control points),

2 - GGD (general global deformation, 49), and

3 - CLD (complex local deformation, 217).

Although model deformations start at the ICA scale (using only 9 control points), the initial distance estimation is done at the GGD scale. This allows for more precise computation of the undirected Hausdorff distance (or the partial distance [4] for noisy sequences) used as a first threshold $\left(T_{1}\right)$ employed by the method. The meaning of this threshold is an estimate of the largest allowed motion in a given experiment (later applied to control points).

The initial analysis used to determine possible correspondences is performed at the ICA scale. Euclidean distances are calculated between the closest model and image points (if the forward Hausdorff distance was larger), or between the closest image and model points (if the reverse Hausdorff distance was larger). These distances are sorted in decreasing order (Fig. 3). $T_{1}$ is then applied to weed out erroneous matches which are possible at any scale. However, at coarse scales, displacements greater than $T_{1}$ are simply infeasible (by definition of the Hausdorff distance).

Another threshold $\left(T_{2}\right)$ is then introduced to deal with erroneous matches resulting in small displacements (results of noise and incorrect matches). At each point we find the slope of the tangent to the curve Displ $=f(i)$, where $\mathrm{i}$ is the index of the correspondences sorted in decreasing order with respect to resulting 3-D displacements $\left(\sqrt{d x_{i}^{2}+d y_{i}^{2}+d z_{i}^{2}}\right)$. When an absolute value of this slope (or function derivative at a point) at least triples (see Fig. 2(c)), the corresponding 3-D displacement value is chosen automatically as threshold $T_{2}$ (usually it increases 4-4.5 times). This threshold can also be computed by using a second derivative or analysis of consecutive differences between (sorted) displacements. $T_{2}$ allows for separation of displacements representing another group of erroneous correspondences, namely, those with small displacements. This group contains a number of wrong matches, especially during the first few iterations (Fig. 2(c)). 


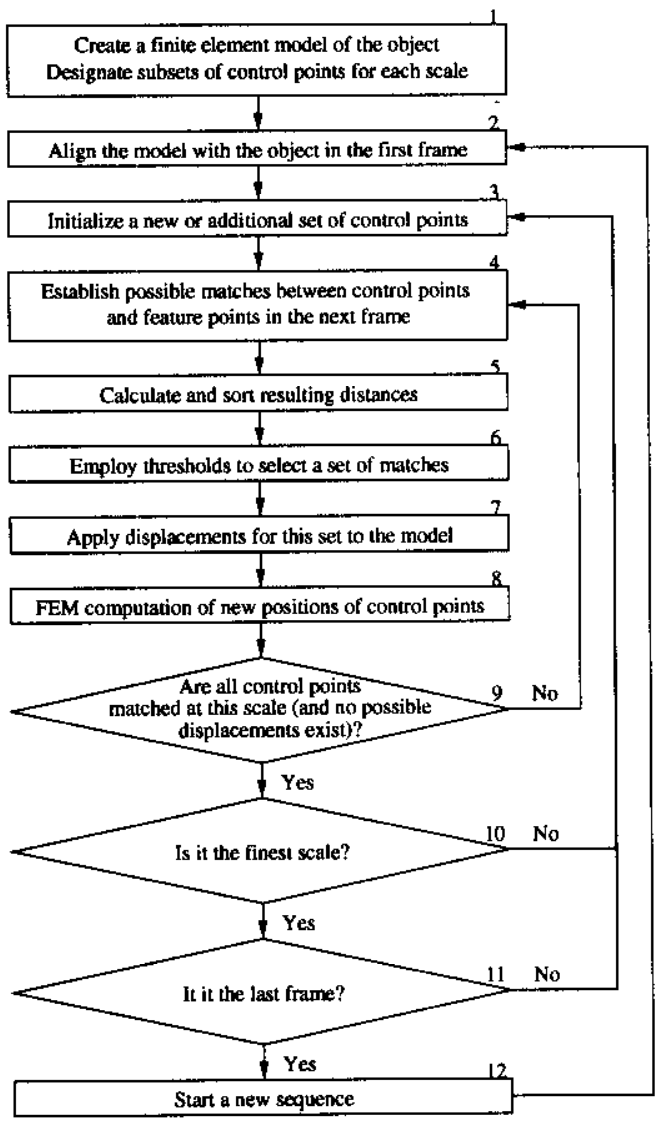

Figure 3: Algorithm of the multiscale approach.

Therefore, a sparse model is employed to select only 1:1 correspondences and discard the rest. Of course, a number of potentially useful correspondences are rejected during this step. This does not matter since at this stage alignment that accounts mostly for translation is more important. Finer model scales process more data that explains nonrigid deformation of the object. If threshold $T_{2}$ cannot be found, then the forward Hausdorff distance (at the coarse scale only) is used to align the model uniformly with the next frame data. It is a good approximation of translation between the frames for the considered subset of nonrigid motion.

A change in scale occurs when a current scale no longer improves the alignment. This means that all control points have been assigned correspondences and there is no mismatch between them and the area of interest in the next frame (Fig. 3). Therefore, increase in model scale at this point produces possibility for improving tracking quality.

GGD (49 control points) and CLD (217 control points) scale processing are similar to the coarse scale iterations, except that $T_{1}$ is not needed. Since finer aspects of object motion are analyzed, concern for filtering out abnormally large displacements is not justified.

The GGD scale addresses effects of elastic motion (stretching) of the object. It results in a better alignment and accounts for most nonrigid deformations. The CLD scale does not improve tracking significantly if the force is distributed along some real or imaginary line/surface rather than represented by a concentrated loading. In the latter case GCD processing alone cannot account for more complex deformations of grid lines. The steps applicable to all scales are shown in Fig. 3.

The model is displacement-driven; when correspondences are established, displacements are calculated and applied to control points of the model. The process is repeated during each iteration. Again, in these experiments, the motion of the object is elastic deformation. At any scale, the process can be summarized as follows:

- The Hausdorff distance is computed.

- For each control point, possible displacements are found and applied.

- The model is incremented accordingly.

- The process iterates until the difference between the model and the object is minimal (for each frame).

\section{Experimental Results}

This section presents an application of the proposed method to motion analysis of man-made elastic materials and human skin. Usefulness of the method is evaluated not only for tracking and motion analysis, but also for a specific application to strain analysis in the burn scar detection procedure.

The model used in experiments described below is local; it covers only a part of the object with the region of interest (the grid in Sections 3.1-3.3 and irregular features in Section 3.4). Second, the model is linear, elastic, and consists of thin elastic shell elements defined in a 3-D space (a total of 324 elements and 361 nodes). The same mesh and solution are used in all experiments described in this section. For more details on model-building, finite element calculation, implementation using ANSYS package and skin parameter selection (material properties and thickness), please see [13].

\subsection{Application of the Method to Skin Motion Analysis: A Closer Look at Scales and Iterations}

This subsection presents application of the method to skin motion analysis. The experiment presented in this section addresses our current burn scar assessment research described in [13]. The proposed method substitutes the two previously used separate models such as snakes and FEM. In this section we use images with the grid so that the new method can be compared with an old approach. However, later in Section 3.4 it is shown that the proposed method does not need a grid and can take advantage of the natural features (such as birthmarks). 
One range and two intensity images of a region of skin being stretched containing a burn scar are shown in Fig. 4. The presence of a burn scar contributes to non-uniformity of elastic motion.
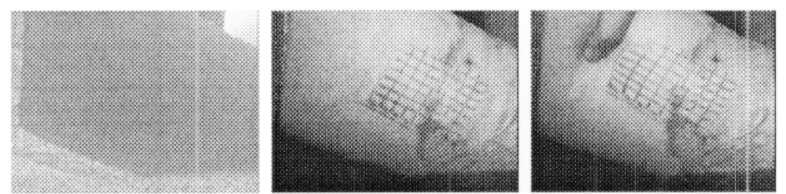

Figure 4: Range and intensity images of skin motion.

Control points of the generic grid model are manually aligned with the first frame using both intensity and range data. Then the method proceeds automatically using the available data, model and the strategy described in Section 2.3. All three defined scales are used. Thresholds are determined using the undirected Hausdorff distance $\left(T_{1}\right)$ and the jump in distances computed between corresponding points $\left(T_{2}\right)$ similarly to Fig. 2(c). The change in scales occurs when all such distances are equal to zero. The solution required a total of seven iterations. Control points of the model are determined automatically and moved as follows (although the grid is slightly rotated clockwise, for the simplicity of explanation we will refer to control points as leftmost and rightmost as if grid lines were vertical):

- iteration 1 - three leftmost control points moving toward the left side of the grid (ICA scale),

- iteration 2 - three rightmost control points moving toward the right side of the grid (ICA scale),

- iteration 3 - correspondences and motion for the remaining model points (ICA scale),

- iteration 4 - motion of new topmost control points (GGD scale),

- iteration 5 - remaining correspondences responsible for general deformation aspects (GGD scale),

- iteration 6 - better approximation of the leftmost line (CLD scale), and

- iteration 7 - other local deformation aspects (CLD scale).

Table 1: Summary of iterations (between two frames in Fig. 4) at different scales.

\begin{tabular}{|l|c|c|c|c|c|c|c|}
\hline Scale & \multicolumn{3}{|c|}{ ICA scale } & \multicolumn{2}{c|}{ GGD acnle } & \multicolumn{2}{c|}{ CLD icale } \\
\hline Iteration & 1 & 2 & 3 & 4 & 5 & 6 & 7 \\
\hline Avg.dist., mm & 7.19 & 3.39 & 0.81 & 0.52 & 0.19 & 0.11 & 0.10 \\
\hline Avg. error, \% & 70.17 & 33.04 & 7.92 & 5.10 & 1.87 & 1.07 & 0.95 \\
\hline
\end{tabular}

Results are shown in Table 1. Both iterations and scales are included. The average real motion between feature points in two frames is 10.253 millimeters (mm). Of course, the motion is not uniform, it is greater for the areas closer to the place where the force is applied. The average distance between control points in the model and corresponding points of the grid is calculated for validation purposes. It is used to compute the average error (a ratio of recovered and real motion of feature points). Performance

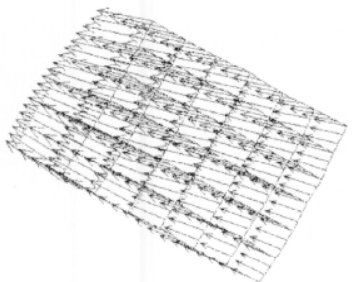

(a)

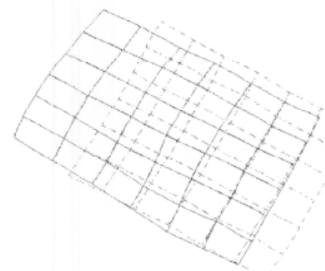

(b)
Figure 5: Results of the skin motion experiment. (a) Magnitude based vectors representing motion of control points. (b) Results in terms of grid motion between two frames.

of the new method was compared to the traditional approach that uses a separate snake model to recover a sparse set of correspondences (grid intersections) and a dense set using FEM model. The new approach produced not only a lower final average error $(0.95 \%$ vs. $1.54 \%$ ), but also a better execution time on a SUN UltraSPARC 300MHz/ 512K cache/128MB RAM (24 seconds vs. 1 minute 7 seconds). The error is reduced because CLD scale better accounts for a non-uniform grid line curvature near the stretching force. The total motion of control points is shown in Fig. 5(a) using magnitude based vectors (CLD scale, last iteration). Red denotes the position of the grid in the previous frame; vectors are displayed in blue. We can visualize grid motion between frames by connecting control points at the finest (CLD) scale (Fig. 5(b)).

\subsection{Results of Motion and Structure Re- covery of Elastic Objects: Perfor- mance Analysis for Longer Sequences}

Sequences of intensity and range images depicting the stretching of an elastic material are utilized for experimental performance assessment of the method. A sequence containing seven intensity images and range images represents input to the algorithm (only one range image is shown in Fig. 6) along with a generic grid model fitted to the initial frame (Fig. 1).

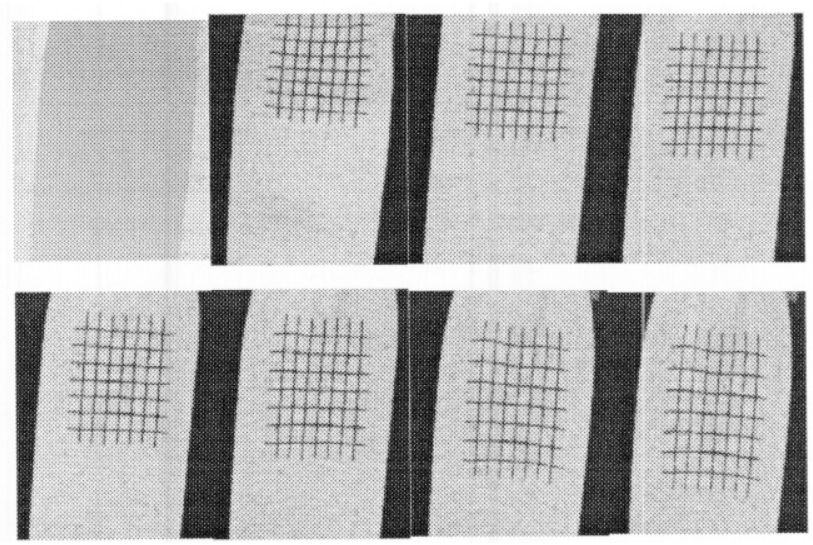

Figure 6: Range and intensity images of the elastic material during stretching.

The deformation is produced by incrementally in- 
creasing the force causing it. The force is introduced in the second frame. It changes from 1 newton $(\mathrm{N})$ in the second frame to $3.5 \mathrm{~N}$ in the last frame in $0.5 \mathrm{~N}$ increments. This sequence allows us to investigate elastic motion in the intervals of material behavior where it can be approximated by a linear, elastic model. Material properties are computed experimentally (using a conventional mechanical engineering technique) and included in the model. Magnitude based vectors representing motion of control points are shown in Fig. 7.
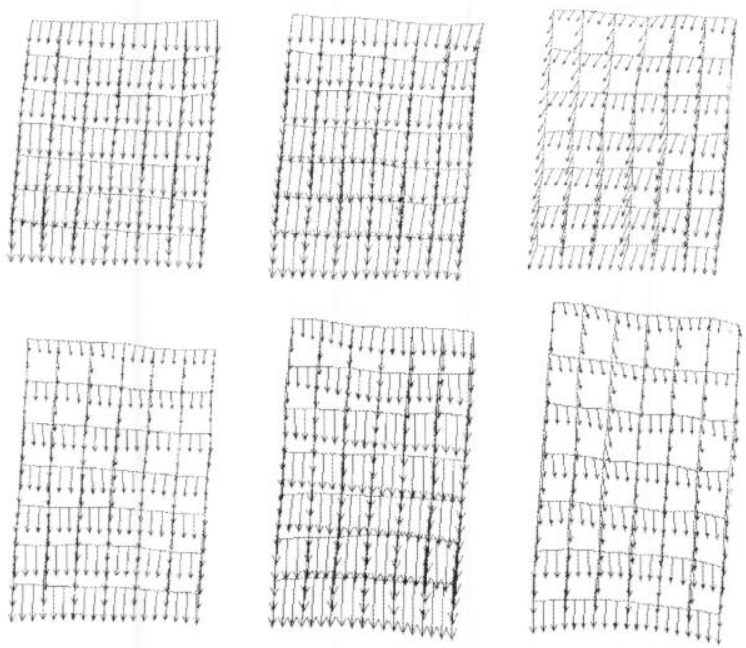

Figure 7: Magnitude based vectors representing motion of control points.

Table 2: Performance of the method for a longer sequence. Results are shown per frame, for final iterations only. Method (1) is a conventional approach (separate snake and finite element models), (2) denotes the proposed multiscale single-model method.

\begin{tabular}{|l|c|c|c|c|c|c|}
\hline Frame number & 2 & 3 & 4 & 5 & 6 & 7 \\
\hline Force, N & 1.00 & 1.50 & 2.00 & 2.50 & 3.00 & 3.50 \\
\hline Real avg.dist., mm & 7.553 & 8.440 & 5.199 & 7.646 & 7.465 & 4.803 \\
\hline Avg.error, \% - (1) & 0.78 & 0.72 & 0.73 & 0.75 & 0.84 & 0.87 \\
\hline Avg.error, \%-(2) & 0.77 & 0.78 & 0.74 & 0.70 & 0.79 & 0.84 \\
\hline
\end{tabular}

Summary of results for all frames are displayed in Table 2. Results are shown per frame, for final iterations only. The proposed approach performs better than the conventional for frames with more complex local deformations where CLD scale or even finer scales are beneficial. Performance comparison for a number of different experiments involving elastic stretching is shown in Table 3 (experiments \#1 and \#2 are ones presented in details in the previous and current subsections, respectively).

Table 3: Performance comparison for a number of different experiments involving elastic stretching.

\begin{tabular}{|l|c|c|c|c|c|}
\hline Experiment & 1 & 2 & 3 & 4 & 5 \\
\hline Avg. error, \% - method (1) & 1.54 & 0.87 & 2.17 & 1.53 & 0.64 \\
\hline Avg. errof, \% - method (2) & 0.95 & 0.84 & 1.30 & 1.19 & 1.72 \\
\hline
\end{tabular}

The number of iterations per frame differs; however, it is on average between 5 and 8 . Linear FEM solution for each iteration takes between 3 and 4 seconds on a UltraSPARC $(300 \mathrm{MHz} / 512 \mathrm{~K}$ cache/128MB RAM). Therefore, solution requires less time (on average 23 seconds) than the old approach (more than 1 minute per frame).

The reason why the number of iterations varies, especially during the ICA step, can be explained with the following observations. The motion seems more or less uniform across all frames; however, analysis of displacements reveals that in the first two frames the grid predominantly translates (since the grid contains only a part of the stretching material), while the remainder of frames contain mostly elastic motion (stretching). Quantitatively it can be described as a ratio of displacements between opposite grid points along the force direction. Ratios close to 1 denote translation, ratios from 3 to 5 in our experiments indicate stretching.

That is why if the next frame is the frame where the bandage mostly translates (for instance, first frame), then the ICA part of the method proceeds faster. This reduction in the number of iterations facilitates the finding of almost all coarse scale correspondences during the first iteration as opposed to 3-4 iterations otherwise.

\subsection{Use of the Method for Strain Analysis}

This subsection demonstrates applicational value of the outlined method for strain analysis. The application addressed here is the computation of human skin response to applied load that reveals differences in underlying properties. For instance, it allows for the detection of burn scars and estimation of their relative properties [13]. Of course, accuracy of the structure and correspondence recovery is very important in such an application because it greatly influences resulting strain distributions that pinpoint differences in properties. Strain is recovered after the last iteration since these differences are detected better using the entire range of motion. Resulting displacements are computed as the differences in positions of control points between the first and the last iterations: $\Delta x_{f i n}=x_{n}-x_{1} ; \Delta y_{f i n}=y_{n}-y_{1} ; \Delta z_{f i n}=z_{n}-z_{1}$. Strain is then recovered throughout the surface of the model [13]. Scars restrict the motion, and, therefore, the method is identifying low strain areas (denoted with dark blue in Fig. 8). The legend column on the right of strain distributions shows (top to bottom): maximum displacement, minimum and maximum strain, and strain gradation from the lowest to the highest. These results correspond to the skin motion experiment (Fig. 4). Fig. 8(a) is obtained with a conventional approach (using separate snake and finite element models, also applied to the available 3-D data [13]). Strain recovered using the proposed method is shown in Fig. 8(b). The resulting strain map is precise enough to identify abnormal areas such 
as scars (ground truth in the form of scar outlines was provided by physicians). A conventional approach identified correctly $93.83 \%$ of the burn scar area; the new method identified correctly $95.68 \%$ of the burn scar area. Methods were compared using five burn scar image sequences. The strain can also be used as an additional criteria to restrict impossible modes of motion.

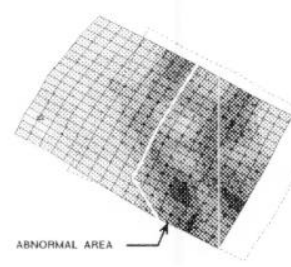

(a)

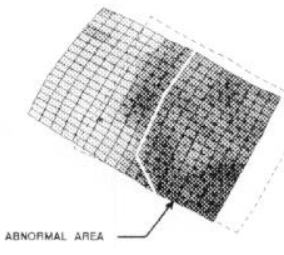

(b)

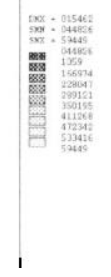

Figure 8: Resulting strain distribution for the skin motion experiment computed using (a) conventional approach and (b) proposed method.

\subsection{Motion Recovery from Irregular and Natural Features}

This section extends the use of the proposed method to deforming objects with irregular surface features. These features cannot be easily considered with a conventional approach. First, another piece of an elastic material (considered initially in Section 3.2) is stretched (Fig. 9). Stretching is non-uniform affecting the upper side of the bandage much more than the lower. In this case model fitting procedure adapts the generic model so that it fits the range data and identifies feature points (shown as small circles). Validation points (shown as crosses) are not used during the computation, the difference in their positions before and after the motion is compared to model's estimates after the process completes. There is no single solution to model fitting. The model is acceptable as long as it contains feature points, uses adequate resolution to represent sensed data, and avoids abrupt changes in the element sizes from very fine to coarse (which can lead to ill-conditioning problems). Resolution scales and threshold selection techniques are the same as in previous experiments. Results (shown in Fig. 10) include magnitude based vectors representing motion of all model points and feature points only. The validation error is less than $3 \%$ for all validation points (Table 4).

Table 4: Motion error for validation points.

\begin{tabular}{|l|c|c|c|c|c|c|c|c|}
\hline Point & 1 & 2 & 3 & 4 & 6 & 6 & 7 & Avg \\
\hline Dist., mm & 3.40 & 3.72 & 7.21 & 7.67 & 8.03 & 12.91 & 15.85 & 8.40 \\
\hline Error, \% & 2.81 & 2.83 & 2.60 & 2.66 & 2.71 & 2.57 & 2.49 & 2.67 \\
\hline
\end{tabular}

A similar experiments are conducted using natural features instead of marked points. Fig. 11(a-c) show intensity and range images of skin stretching. Note that there are no artificial markings on the skin. In
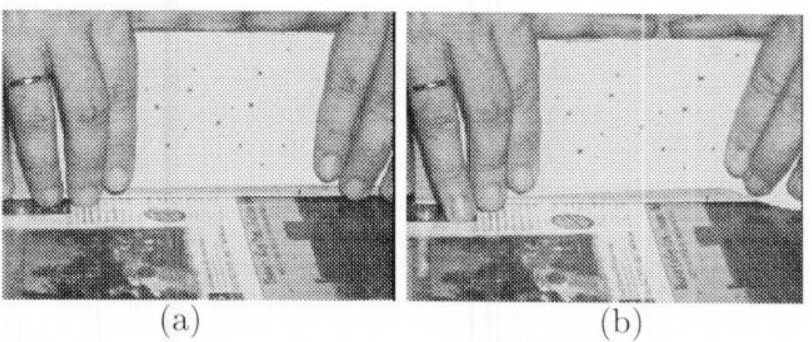

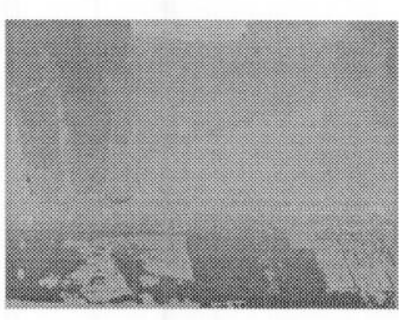

(c)

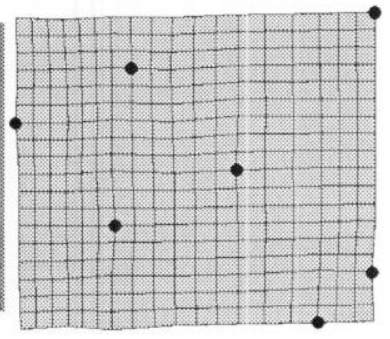

(d)
Figure 9: (a-b) Intensity images of the elastic material before and after deformation. Feature points are marked as small black circles, validation points - as crosses. (c) Range image (before motion). (d) Finite element model (in terms of elements) fitted to the data. Feature points are identified.

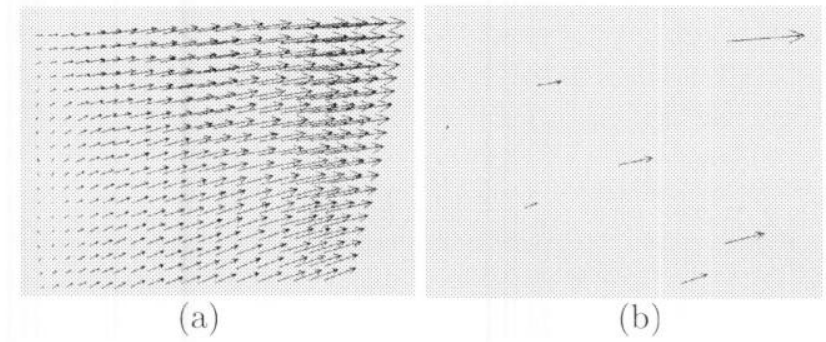

Figure 10: Magnitude based vectors representing motion of (a) all model points and (b) feature points only.

this case birthmarks are chosen as features to include into the model (similarly to feature points in the previous experiment) and to use for motion analysis (resulting displacement fields for them are displayed in Fig. 9(d)). Six points are used for validation (the average error is $4.72 \%$ ). This shows that the method can be extended to other applications and domains, and simplify data acquisition and processing for many existing applications (such as a burn scar assessment application briefly described in Section 3.3).

\section{Discussion and Conclusions}

In this paper we presented a novel multiscale approach to recovery of nonrigid motion from sequences of registered intensity and range images. The main idea of our approach is that a finite element (FEM) model can naturally handle both registration and deformation modeling using a single model-driving strategy. The method includes a multiscale iterative algorithm based on analysis of the undirected Hausdorff distance to recover correspondences. Our model can 


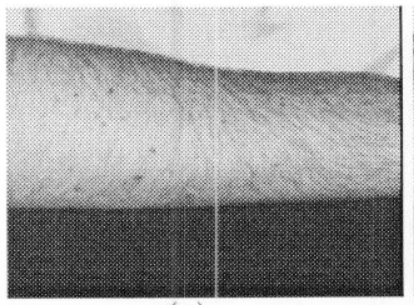

(a)

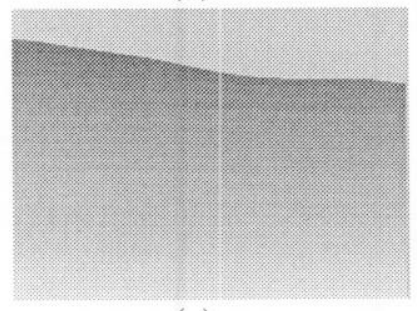

(c)

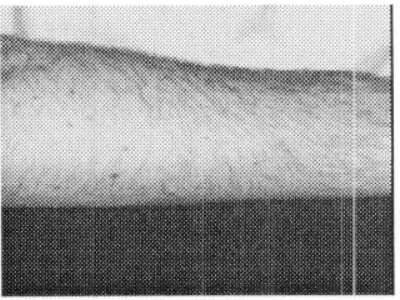

(b)

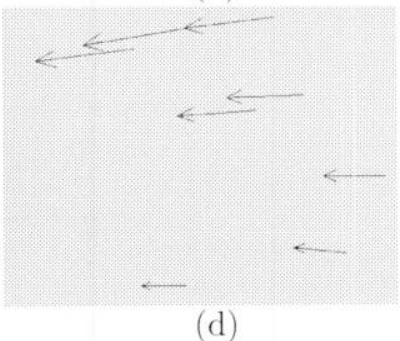

Figure 11: (a-b) Intensity and (c) range images of skin stretching. (d) Motion tracking of feature points.

handle what previously was accomplished using two types of deformable models (snakes and finite element models). Control points used for tracking are also a part of the finite element model containing knowledge of an object's properties that can lead to better analysis of the deformation process. Therefore, not only intersection points, but also additional tracked points are included in the model. Such a model can explain observed motion effects (such as displacements) as well as non-observable aspects (such as strains). Strain distributions reveal differences in material properties which can account for motion abnormalities.

The method includes a multiscale strategy based on evaluation of the undirected Hausdorff distance which represents a reliable error function. Wrong matches occur, but they are corrected during subsequent iterations. In a general case, a number of scales is objectand motion-dependent, similarly to other physicallybased models. Choosing the number of control points at the finest scale is a trade-off between the efficiency of structure representation and effects of noise. (It has been found experimentally that for our setup the scanning error is between $0.5 \mathrm{~mm}$ and $1 \mathrm{~mm}$.)

The method was evaluated with respect to speed, accuracy, and noise sensitivity. Advantages of the proposed approach were demonstrated using man-made elastic materials and human skin motion. Experiments with regular grid features were used for performance comparison with a conventional approach (separate snakes and FEM models). It was shown, however, that the new method does not require a grid and can adapt the model to available object features. Usefulness of the method was presented not only in the context of tracking and motion analysis, but also for specific applications such as burn scar detection. This work presents a significant step toward development of models that can inherently handle multiple processing functions, currently registration and deformation, and appearance in the near future.

\section{Acknowledgment}

We would like to thank Alok Gupta (Siemens Corporate Research) for an invaluable discussion with us on hybrid models during CVPR'98.

\section{References}

[1] A. A. Amini, Y. Chen, R. W. Curwen, V. Mani, and J. Sun. Coupled B-snake grids and constrained thin-plate splines for analysis of $2 \mathrm{D}$ tissue deformations from tagged mri. IEEE Trans. on Med. Imag., 17(3):344-356, June 1998.

[2] S. Basu and A. Pentland. A three-dimensional model of human lip motions trained from video. In IEEE Nonrigid and Articulated Motion Workshop, pp. 4653, San Juan, PR, June 1997.

[3] S. Chandran and A.K. Potty. Energy minimization of contours using boundary conditions. IEEE Trans. on PAMI, 20(5):546-549, May 1998.

[4] D. P. Huttenlocher, G. A. Klanderman, and W. J. Rucklidge. Comparing images using the Hausdorff distance. IEEE Trans. on PAMI, 15(9):850-863, September 1993.

[5] S. Kumar and D. B. Goldgof. Automatic tracking of SPAMM grid and the estimation parameters from Cardiac MR images. IEEE Trans. on Med. Imag., 13(1):122-132, March 1994.

[6] J. Martin, A. Pentland, S. Sclaroff, and R. Kikinis. Characterization of neuropathological shape deformations. IEEE Trans. on PAMI, 20(2):97-112, Feb 1998.

[7] J. C. McEachen, II, and J. S. Duncan. Shape-based tracking of left ventricular wall motion. IEEE Trans. on Med. Imag., 16(3):270-283, June 1997.

[8] J. Montagnat and H. Delingette. A hybrid framework for surface registration and deformable models. In Proc. CVPR, pp. 1041-1046, PR, June 1997.

[9] T. O'Donnell, T. Boult, and A. Gupta. Global models with parametric offsets as applied to cardiac motion recovery. In Proc. CVPR, pp. 293-299, June 1996.

[10] J. Park, D. Metaxas, A. Young, and L. Axel. Deformable models with parameter functions for cardiac motion analysis from tagged MRI data. IEEE Trans. on Med. Imag., 15(3):278-289, June 1996.

[11] A. Singh, D. B. Goldgof, and D. Terzopoulos (editors). Deformable Models in Medical Image Analysis. IEEE Computer Society Press, Los Alamitos, CA, 1998.

[12] H. Tao and T. S. Huang. Connected vibrations: A modal analysis approach to non-rigid motion tracking. In Proc. CVPR, pp. 735-740, CA, June 1998.

[13] L. V. Tsap, D. B. Goldgof, S. Sarkar, and P. Powers. A vision-based technique for objective assessment of burn scars. IEEE Trans. on Med. Imag., 17(4):620633, August 1998.

[14] B. C. Vemuri and A. Radisavljevic. From global to local, a continuum of shape models with fractal priors. In Proc. CVPR, pp. 307-313, NY, June 1993.

[15] A. Young, D. Kraitchman, L. Dougherty, and L. Axel. Tracking and finite element analysis of stripe deformations in magnetic resonance tagging. IEEE Trans. on Med. Imag., 14(5):413-421, September 1995. 